\title{
L'enseignement du français au Maroc, trêve de relativisme culturel
}

\section{Soumya El-Harmassi}

\section{(2) OpenEdition \\ 1 Journals}

\section{Édition électronique}

URL : http://journals.openedition.org/trema/155

DOI : 10.4000/trema.155

ISSN : 2107-0997

\section{Éditeur}

Faculté d'Éducation de l'université de Montpellier

\section{Édition imprimée}

Date de publication : 1 novembre 2008

Pagination : $39-48$

ISSN : 1167-315X

\section{Référence électronique}

Soumya El-Harmassi, « L'enseignement du français au Maroc, trêve de relativisme culturel », Tréma [En ligne], 30 | 2008, mis en ligne le 01 novembre 2010, consulté le 19 avril 2019. URL : http:// journals.openedition.org/trema/155; DOI : 10.4000/trema.155

Ce document a été généré automatiquement le 19 avril 2019.

Trema 


\title{
L'enseignement du français au Maroc, trêve de relativisme culturel
}

\author{
Soumya El-Harmassi
}

1 Après plusieurs années de discours alarmistes sur le niveau de l'enseignement au Maroc, on parle volontiers, depuis la publication de la Charte Nationale d'Éducation et de Formation en 1999, d'un renouveau possible. Les notions de "qualité», voire « d'excellence » sont omniprésentes dans les titres des innombrables colloques et autres congrès, organisés aussi bien par le Ministère de l'Éducation Nationale, les Académies et les Universités que par différentes associations d'enseignants intégrés à des réseaux internationaux d'«experts ». Le chantier de la décennie que constitue la Réforme a, incontestablement, impulsé une dynamique de réflexion sans précédent.

2 Il nous semble néanmoins qu'un décalage existe entre ce vaste mouvement de manifestations officielles, le foisonnement apparent d'idées et de recommandations et la force intellectuelle potentielle en attente d'orientation, de structuration, d'enrichissement, d'ouverture que l'on perçoit chez les enseignants, chez les élèves et chez les étudiants.

3 Emportés par le charme équivoque des échafaudages théoriques proposés par une « modernité » soucieuse de construire une « économie » planétaire de l'enseignement et de la formation, nous importons, en les mimant, des orientations didactiques et des programmes pédagogiques sans que soient entreprises des recherches préalables d'adaptabilité. Les questions liées à l'enseignement des langues et de la littérature telles que la délimitation des savoirs culturels ou l'utilité de l'enseignement de la littérature, qui impliquent des interrogations philosophiques sur la place que doit occuper l'individu dans son environnement social, économique et politique, sont soigneusement passées sous silence. Les discours officiels feignent d'ignorer la nature du terrain et évitent le travail d'ajustement à entreprendre en amont des réformes. Cette incapacité à dépasser des paradoxes "convenus" freine toute dynamique de recherche et contraint les enseignants à pérenniser les modèles existants, d'autant que la réforme n'a pas véritablement prévu de plan de formation continue ni même de refonte de la formation initiale. Les nouveaux programmes, voués à se plier à des habitudes de travail 
antérieures, ont été adoptés sans aucun signe de contestation. L'action pédagogique s'est ainsi trouvée, de nouveau, d'un côté, figée en une raideur doctrinale décalée et, de l'autre, grevée d'automatismes lourdement ancrés aux confins de l'aire difficilement pénétrable où les choix éducatifs croisent le social et le politique en un jeu informel, complexe et, néanmoins, décisif.

Prenons l'exemple de l'enseignement des langues dans le cycle secondaire qualifiant et, en particulier, celui du français.

Et partons du bilan publié par la Commission Spéciale Éducation - Formation dans le rapport qui clôture son mandat et qui contient une évaluation détaillée des quatre premières années de la Réforme ${ }^{1}$.

La question des langues est communément reconnue comme un facteur structurel de la crise de l'école en raison de l'incohérence des choix qui ont longtemps prévalu aussi bien en matière de "langues d'enseignement " qu'en matière "d'enseignement des langues"; des choix qui se sont traduits le plus souvent par une faible maîtrise des langues par les élèves et les étudiants. Que ce soit la langue arabe, ou les autres langues étrangère, le niveau linguistique des étudiants reste globalement faible tant au niveau de l'écrit que de l'expression orale. [...]

Deux problèmes importants demeurent particulièrement préoccupants: d'une part le déphasage linguistique entre le secondaire où l'essentiel des enseignements se fait en langue arabe et le supérieur où l'arabisation n'a concerné qu'une partie des sciences humaines à l'exclusion des autres champs scientifiques, technologiques et professionnels, d'autre part entre la langue d'enseignement qui est l'arabe et les langues exigées dans la vie professionnelle.

6 La crise de l'école est formellement reconnue et elle est, en ce qui concerne son versant pédagogique, officiellement reliée à l'incohérence des choix ayant prévalu en matière d'aménagement linguistique scolaire.

Cet acte de reconnaissance officielle est, en soi, une véritable révolution.

8 Reste à analyser les réalisations effectives des orientations pédagogiques prônées par la réforme ...

Deux principes innovants majeurs structurent les nouvelles recommandations: l'enseignement du français par l'œuvre littéraire ${ }^{2}$ et la pédagogie par compétences. Ces deux lignes de force, que rien ne relie a priori, se justifient en raison de la prédominance, au niveau des discours officiels, de deux matrices disciplinaires imposées par les projets de "mondialisation" de la formation: "l'ouverture culturelle», et la littérature constitue une mémoire patrimoniale incontestable, et "l'efficience» en matière de savoirs professionnels, identifiable à partir de grilles de connaissances inductives, définies sur la base de modèles préformés de " compétences ".

10 Le fait est qu'après près de cinq années d'application des nouveaux programmes, les évaluations et les tests effectués aux différents niveaux et cycles du système attestent toujours d'une faible maitrise des langues ${ }^{3}$.

11 Il nous semble que ce déficit en compétences linguistiques ${ }^{4}$ est, en grande partie, imputable, non pas tant au caractère « inadapté » des choix pédagogiques effectués, qu'à ce que nous nous permettrons d'appeler "une force centripète d'acclimatement". L'absence d'une dynamique de réflexion et de recherche, émanant de pratiques pédagogiques effectives, met les enseignants dans un état de fragilité intellectuelle tel qu'ils en sont réduits à adapter, de façon isolée, les consignes qui leur sont données, servant ainsi toutes sortes d'instrumentalisations. L'éviction de la littérature au profit des 
« documents authentiques » est, à ce propos, un exemple significatif. Elle fut justifiée au nom d'un argument anthropologique diffus : la substance culturelle des textes serait trop éloignée des représentations des élèves et entraverait, de ce fait, la maîtrise de la langue. Le même motif sert désormais un choix strictement contraire : le retour de la littérature garantit une meilleure maitrise de la langue, mais il est nécessaire de standardiser le corpus des œuvres au programme à l'échelle nationale. Aucune preuve scientifique n'a été construite afin d'entériner l'un ou l'autre choix. L'énoncé intuitif d'une diversité de modèles culturels, indéfinis, suffit. La littérature charrierait des codes moraux et des mœurs sociales susceptibles de brouiller "l'identité nationale", on en assure le « contrôle » par l'unification du programme des œuvres.

Des stratégies implicites de résistance au changement structurent le terrain et entravent le processus d'installation et de mise en œuvre, efficace, des réformes. Seul un travail de recherche multidisciplinaire permettrait de les définir. Risquons quelques hypothèses.

Et commençons par « la pédagogie par compétences ».

ous les textes des recommandations pédagogiques ${ }^{5}$, par discipline, lui consacrent un long paragraphe qu'ils déclinent en rubriques cloisonnées : les compétences stratégiques, les compétences communicatives, les compétences méthodologiques, les compétences culturelles et les compétences technologiques. Cette fragmentation, qui se présente comme un gage de rationalité, induit, en réalité, un mode de transfert réducteur par lequel la cohésion recherchée est neutralisée au nom d'un «scientisme » destiné à sauvegarder l'immuabilité d'une "culture» d'où l'expression individuelle et l'esprit critique sont bannis. Faire des compétences l'enjeu majeur de l'apprentissage, les définir, les sérier en référence à des «familles de tâches - problèmes » écrites ou orales, autorise une autre forme de standardisation : la systématisation resserrée, uniformisante, exclut les savoirs non évaluables et les stratégies individuelles d'accès au sens.

Un rapide coup d'œil jeté aux compétences clés prônées dans le cadre de référence européen, considérées comme nécessaires à tous en ce qu'elles fondent l'épanouissement personnel, l'inclusion sociale, la citoyenneté active et l'emploi, et les thèmes y afférant, la réflexion critique, la créativité, l'esprit d'initiative, la résolution de problèmes, l'évaluation des risques, la prise de décision et la gestion constructive des sentiments, permet de souligner qu'un postulat de base est à l'œuvre dans le cadre européen : la liberté d'expression et d'action individuelles.

16 L'esprit de veille idéalement visé par ces approches est, de toute évidence, contrôlé, modéré, en une subtile complexité, par le nécessaire travail de nivellement accompagnant, en général, tout programme éducatif. Il n'en demeure pas moins qu'elles semblent reposer sur un principe cohésif inédit : la formation à une gestion autonome du rapport au savoir et au monde censée garantir à chacun l'articulation de trois facettes jusque là distinctes, la culture, la vie et le métier. Est ainsi postulée, ce qui est à l'horizon des grilles les plus formellement judicieuses, cette sagesse aux effets différés qui permet aux individus de garder les yeux ouverts.

17 C'est que la pierre angulaire sur laquelle s'articule la philosophie inhérente à ce choix de formation, du moins à un niveau théorique, est indissociable de la culture humaniste que l'Occident a édifiée au cours de plusieurs siècles et au rythme ininterrompu de révolutions et de contre - révolutions artistiques, philosophiques, sociales et politiques. La confiance exaltée dans les facultés humaines a été longuement mûrie. La sacralisation de l'écrit et du point de vue unique, en ce qui concerne les codes de délimitation des 
valeurs (le bien et le mal, le vrai et le faux, le juste et l'injuste, l'acceptable et le non acceptable dans l'aire des savoirs), en a été définitivement dépassée. L'établissement, par les philosophes, les poètes et les historiens, de rapports identifiables entre le progrès, l'évolution des mœurs et la création individuelle est, au moins depuis les Lumières et les premiers romantismes, tributaire de l'émergence d'un sujet libéré des règles, maître de son regard et de sa pensée. Un long, et parfois violent, bouleversement s'est opéré dans le champ des savoirs qui a annoncé la libération de la subjectivité des contraintes sociales, des idéologies et des mythologies. Il a profondément travaillé ${ }^{6}$ les consciences individuelles et collectives, sociales et politiques.

Et voilà que nous sommes invités, au Maroc, à réaliser, très rapidement, de façon purement technique et à l'intérieur d'un environnement culturel incompatible, la même coupure...

Le développement des compétences clés est tributaire d'un travail d'individualisation et de socialisation qui dépasse largement le strict cadre de l'école. L'étude des oeuvres gagne à être assortie d'une richesse et d'une diversité des pratiques culturelles, telles que la lecture ou la fréquentation de lieux de culture, afin d'aider réellement au développement des capacités réflexives. Le foisonnant réseau de création et de réception culturelles et idéelles mis à la disposition de l'élève européen est, ici, totalement absent. Le contexte social, sclérosé, est en rupture nette avec « la culture scolaire ».

Il est un fait désormais incontestable : la pensée et la culture occidentales se sont très tôt libérées de leur ancrage mytho - théologique, les «intellectuels», portés par un élan prophétique ou " pro gnostique ", y ont assuré définitivement l'individu que son pouvoir créateur est lié à sa liberté « subjective ».

21 Ce n'est pas exactement le cas de la culture arabe. Les travaux de recherche de penseurs modernes comme Abdallah LAROUI ou Mohammed ARKOUN ${ }^{7}$ le certifient de façon indubitable.

Les conséquences de ce décalage historique et philosophique ne sont pas négligeables. Des travaux de recherches relevant de la littérature et de la linguistique comparées, de la psychologie historique et de l'anthropologie politique devraient être entrepris, qui nous permettront d'en mesurer l'ampleur et d'en sublimer les effets afin de réajuster les formations initiale et continue des formateurs, phase capitale du processus, totalement passée sous silence par la Réforme.

23 Car il s'agit de réduire la teneur de la tentation techniciste, réductrice et fragmentaire qui freinera inévitablement toute réforme induisant l'affirmation d'un mode de pensée individuel, structuré selon les principes d'autonomie et d'innovation.

24 Au niveau de l'enseignement supérieur et de la formation, l'étouffement culturel, historique, de la subjectivité est sans aucun doute responsable de la prohibition, quasi morale, de toute manifestation d'une pensée critique individuelle au profit d'usages ritualistes, profondément ancrés, destinés à maintenir et à consolider les rapports « traditionnels » entre pouvoir et savoir.

Il se trouve que pendant plusieurs décennies, la négation philosophique de l'individu a coïncidé, en Lettres et en Sciences humaines du moins, avec, sur le plan académique, l'éviction du sujet. L'ascèse méthodologique que cela supposait a fait les beaux jours de l'Université marocaine. Il a été possible de se protéger sous le parapluie sécurisant de structures formelles reconnaissables. La fièvre des classements a gagné même le lycée sous la forme de « tabulations », vulgarisation caricaturale des procédures structuralistes. 
26 La course aux systématisations laborieuses imposée par une ère soucieuse avant tout d'édifier des absolus théoriques est désormais dépassée, les discours post-modernes ont réhabilité le sujet et les politiques éducatives se conforment à ces évolutions.

Embarrassés, mal à l'aise, nous développons malgré nous des stratégies de protection contre les désordres que pourrait causer l'implication effective, perçue comme nécessairement subversive, des individus dans un processus d'émancipation et d'ouverture. C'est sans doute la raison pour laquelle plusieurs filières, dans les facultés des lettres et des sciences humaines, se sont engouffrées, sans aucune précaution, dans la brèche ouverte par les théories dites de la "communication ». Les titres des modules, dans leur étonnante décomposition, témoignent de la même propension au technicisme dogmatique. En langue et littérature françaises, l'étude des textes est réduite à un discours objectivant et formaliste: repérer les catégories grammaticales, dresser des inventaires de figures de style (listes réduites, canoniques, retenues de façon automatique). L'esprit d'initiative est ainsi naturellement contrecarré par l'adhésion aveugle, parfois exaltée, à des canevas méthodologiques de qualité inégale. La sensibilité à la diversité culturelle, nécessaire au recul créatif, est même parfois freinée par des grilles de lecture moralisatrices. Les qualités intellectuelles, indispensables à l'implication permanente de chacun dans sa propre formation, sont, en conséquence, affaiblies et la maîtrise de la langue s'en trouve négligée. La force de «dés - implication» est telle qu'elle est imputable, de façon quasi indifférenciée, aux formateurs et aux étudiants. Ni les uns ni les autres ne sont disposés à s'engager dans une logique qui excèderait les ressources codifiantes, classificatoires et systématiques des discours préalables et des "méthodes", pour une participation, "sympathique ${ }^{8}$, quels que soient les risques encourus, à une entreprise, réfléchie, structurée, d'appropriation.

Cet enfermement généralisé est comme transmué en une nécessité à l'aide de procédés qui en assurent la cohérence interne. Les programmes, les modalités d'évaluation, la planification matérielle des enseignements prennent infailliblement le pli ...

C'est ainsi que lors des «modules » consacrés à l'analyse et à la synthèse, y compris (surtout) dans les formations de haut niveau ${ }^{9}$, un temps non négligeable est consacré, à la demande des étudiants, et de façon répétitive, à la mise en place de "grilles » méthodologiques canoniques. C'est ainsi que les débats d'idées ayant caractérisé une époque et qui justifient les grandes scansions historiques d'une civilisation sont réduits à une juxtaposition de " courants ». C'est ainsi que la mobilisation cohérente et raisonnée des connaissances en vue de la construction d'un sens, qualité requise quel que soit le type de formation en Lettres et sciences humaines, se trouve irrémédiablement détournée au profit de la primauté accordée à l'instauration de méthodes. Et la résistance à adopter un point de vue critique, la méfiance vis - à - vis de toute activité induisant une démarche personnelle sont manifestes, conscientes, parfois clairement exprimées. De " nouvelles méthodes » sont, étrangement, générées régulièrement par un forum occulte, lointain, garant de par le mythe qui l'entoure, de leur modernité; et elles sont neutralisées et remplacées de façon périodique comme pour alimenter la soif positiviste d'enseignants en sécurité dans leur rôle de simples exécutants.

31 Comment, dans ces conditions, parvenir à transmettre l'idée selon laquelle le plaisir personnel dans l'assimilation et le dépassement des savoirs n'est pas exclusif de l'adhésion aux normes, et qu'il peut même en favoriser le respect? 

modèle escompté par des réformes fondées sur le souci de rejoindre une modernité qui fait de l'épanouissement de l'individu une nécessité économique, alors même que nous évoluons dans un environnement d'où le « je » est banni et où l'autoritarisme est présent jusque dans l'usage et l'apprentissage des langues?

Quelques voies, qui doivent être empruntées en matière d'enseignement et de formation de façon progressive et en commençant par les cycles supérieurs, sont susceptibles de favoriser un « franchissement " possible de ces impasses :

- l'accroissement de la responsabilité de l'enseignement des langues et des lettres ${ }^{10}$ au niveau du système éducatif d'ensemble ;

- la redéfinition du découpage traditionnel en matières ou en départements : l'enseignement des lettres arabes et françaises, l'enseignement en arabe et en français sont probablement des atouts dont il faudrait tirer profit à l'aide d'aménagements transdisciplinaires ;

- la diversité sémiotique des textes arabes et français, et leur complexité intertextuelle devraient être exploitées, la perception interne des disciplines en serait nécessairement enrichie. L'enseignement des langues et des lettres arabe et française, au lieu d'être cloisonné, pourrait faire l'objet de travaux communs d'homogénéisation des approches, l'enseignement des sciences en langue arabe pourrait être assorti d'un programme de renforcement, formellement établi, intégré au cours de français... ;

- la prise en compte et le réajustement de la réalité des représentations des langues en présence : l'arabe classique est l'idiome porteur de la loi religieuse et du discours politique, la langue maternelle ; le dialectal ou la darija, perpétue une tradition orale dévalorisée en milieu scolaire (et médiatique) ; et le français demeure, quoique les textes officiels veuillent le noyer dans un cumul théorique de « langues étrangères », une part importante de ce faisceau de langues. Cette complexité linguistique recouvre, paradoxalement, un multiculturalisme stratifié, tourmenté. Il s'agit simplement de le reconnaître et d'en rentabiliser les effets grâce à un projet culturel visant l'équilibre et l'harmonie et instaurant des modes d'appréciation consentie et volontaire, gérés par les acteurs eux-mêmes.

sans doute ainsi inaugurée une entreprise dépassionnée, structurée, de décloisonnement, de raccordement serein, de différentes facettes culturelles jusque là en situation de conflit. Serait, en conséquence, assaini le rapport aux langues et garantie, voire consolidée leur maîtrise. Plus que des significations partagées, la culture correspondrait à une véritable posture ontologique acceptant en son sein de multiples postulations. Ne s'agit-il pas avant tout d'intégrer ses altérités propres?

Une réévaluation de la lecture des textes littéraires dans une perspective transdisciplinaire, c'est-à-dire sur la base d'un travail d'homogénéisation minimale des approches $^{11}$, doit constituer, selon nous, le moteur le plus important de cette mutation. Car il s'agit à la fois, d'affirmer la portée universelle de la littérature et de la réinvestir de la mission qui est la sienne : perpétuer l'exigence d'une parole libérée de l'emprise des embrigadements et des carcans idéologiques, maitresse de ses convictions intimes. L'unité des principes de base qui règlent les consignes, ou les canevas méthodologiques, devrait permettre de secondariser les techniques au profit de la combinaison, 
nécessairement enrichissante, des savoirs littéraires acquis en français et en arabe et de renforcer, chez l'étudiant, ou chez l'élève, une posture critique individuelle.

Pour ce faire, deux principes prioritaires devraient être posés préalablement aux programmes de formation en langues et littératures arabe et française :

- Libérer l'enseignement littéraire de la chape techniciste qui l'extériorise : renvoyer les grilles formelles préétablies au statut d'instruments d'études préliminaires ${ }^{12}$, au lieu d'en faire, comme cela est le cas actuellement, une fin en soi, ce qui neutralise les effets de la réception, qu'ils soient d'ordre cognitif, esthétique ou affectif ;

- Inscrire l'entreprise critique dans l'aire intertextuelle, élargie, qu'elle suppose, afin de la protéger des dérives anecdotiques, faussement idéologiques ${ }^{13}$.

La littérature n'est pas l'illustration de canevas préconstruits. Le texte vaut par sa rupture avec les conventions du moment et c'est à cette condition qu'il est intégré dans le champ de l'expérience esthétique, c'est-à-dire qu'il acquiert le statut d'oeuvre classique. En outre, les critiques littéraires et les auteurs l'ont suffisamment démontré, la littérature travaille sur des langages déjà constitués, les œuvres renvoient moins au réel qu'aux autres œuvres. Leur lisibilité dépend donc d'un dialogue intertextuel plus ou moins explicite. "Plus ou moins tous les livres contiennent la fusion de quelque redite comptée », écrivait MALLARMÉ.

Ces deux dimensions de la littérarité, le double fait que le texte précède les artifices théoriques et qu'il se soucie peu de sa fonction documentaire, impliquent un travail d'analyse autrement plus ardu que le simple inventaire des champs lexicaux ou des figures de style utilisées, établi sans obligation aucune de recherche synthétique des effets produits. Il suppose des stratégies pédagogiques tournées vers l'élargissement permanent des lectures personnelles.

Peut-être nous faut-il aussi explorer le postulat selon lequel la réception, la perception des textes littéraires français ou arabe lus par les enseignants et par les élèves est guidée par leur fréquentation des deux littératures? Qu'ils soient autorisés à mobiliser des démarches multiples et des stratégies diverses de représentation, à soumettre intelligemment les textes à des éclairages réciproques. Il revient à l'enseignant qui aura bénéficié d'une formation de haute exigence, d'instaurer des modes structurés, riches, intellectuellement valides de sélection.

Un travail interdisciplinaire est à entreprendre aux niveaux de la création des programmes, de la réflexion sur la formation et de l'enseignement secondaire, qui permettrait d'édifier un socle commun de connaissances et de définir des instruments non exclusifs de lecture, de telle sorte que soit enclenché le processus de modification des horizons, initiateur d'une authentique culture d'ouverture.

41 Force est de reconnaître que, pour l'heure, « les bibliothèques intérieures » de nos élèves et de nos étudiants sont d'une vacuité désolante. Aucun effort n'est jamais consenti pour la création de bibliothèques alors même que des projets pharaoniques sont entrepris pour la généralisation de l'usage des moyens technologiques...

42 Cette méfiance calculée à l'égard du livre a, sans aucun doute, des raisons relevant de registres politiques ou sociaux que nous ne nous hasarderons pas à décrypter. Nous nous limiterons à noter qu'elle rejoint une certaine forme d'ambiguïté délibérément entretenue vis-à-vis de la culture « occidentale ».

Dans les recommandations pédagogiques officielles françaises ${ }^{14}$, il est clairement souligné que L'enseignement du français participe aux finalités générales de l'éducation au lycée / ... / . Il 
contribue à la constitution d'une culture par la lecture de textes de toutes sortes, principalement d'oeuvres littéraires significatives. Il forme l'attention aux significations de ces oeuvres, aux questionnements dont elles sont porteuses et aux débats d'idées qui caractérisent chaque époque, dont elles constituent souvent la meilleure expression. Par là, il permet aux lycéens de construire une perspective historique sur l'espace culturel auquel ils appartiennent. Et, plus loin, il apporte à la formation du citoyen, avec la connaissance de l'héritage culturel, la réflexion sur les opinions et la capacité d'argumenter.

Les textes officiels marocains se limitent à justifier le recours aux textes littéraires par le fait qu'ils garantissent l'acquisition progressive d'un savoir encyclopédique, littéraire et culturel ... pour une meilleure connaissance de la langue cible et l'acquisition de savoirs instrumentaux permettant la résolution de problèmes de lecture et d'interprétation de divers types de textes essentiellement littéraires ${ }^{15}$.

Les rédacteurs de ce document n'ignorent sans doute pas que le texte littéraire n'est pas le dépositaire de l'usage le plus juste et le plus parfait de la langue puisqu'il se définit justement par sa propension à en dénoncer les infirmités, voire l'inauthenticité ou le leurre, par toutes sortes de transgressions (ou de transmutations...). La littérature est, certes, en relation avec le langage mais la logique qui les lie n'est ni descriptive ni expressive. Les œuvres littéraires constituent sans doute la meilleure expression des débats d'idées, elles sont porteuses d'effets esthétiques qui les haussent bien au-dessus du constat platement commun que la littérature est un art langagier, un art des mots. Plus que le perfectionnement langagier (la maîtrise de la langue est la condition première d'accès aux textes, elle doit donc être en partie déjà là), elles permettent aux élèves de développer leur curiosité et de nourrir leur imaginaire. Aucune place n'est laissée à ces deux vecteurs de la pensée dans les documents officiels marocains, la littérature étant renvoyée à une fonction exclusivement instrumentale et patrimoniale ...

Les enseignants $d u$ secondaire sont appelés à user du texte littéraire, il faut qu'ils apprennent d'abord à en jouir ${ }^{16}$. Car, disons - le, même si cela doit paraitre évident, le plaisir de lire s'acquiert et se transmet et il constitue indéniablement la source première et continue de l'esprit de découverte et du contrôle actif des connaissances ${ }^{17}$.

\section{BIBLIOGRAPHIE}

Rapport de la COSEF sur la mise en œuvre de la réforme du système d'éducation et de formation (1999-2004). Journées de formation des professeurs de français, Ministère de l'Éducation Nationale, juin 2002. ADAM, J.-M. (1996), Les Textes, types et prototypes, Nathan Université, 3e édition. ARKOUN, M. (2006), Humanisme et Islam, Librairie philosophique J. Vrin, éd. Marsam. L'Humanisme arabe au IV/Xe (1970), Paris Vrin. La Pensée arabe (2003), Paris, PUF, 6e édition.

LAROUI, Abdallah (1974), La Crise des intellectuels arabes, traditionalisme ou historicisme, Paris, La Découverte. / Islam et Histoire (2000), Albin Michel. / Islam et modernité, 2002, La Découverte. BOURDIEU, P. (1992), Les Règles de l'art, Seuil. 
CHISS, J.-L. (1995), Didactique du français, état d'une discipline, Nathan Pédagogie.

KERBRAT ORECCHIONI, C. (1986), L'Énonciation, Armand Colin.

LEGENDRE, P. (1994), Dieu au miroir. Étude sur l'institution des images, Fayard.

TODOROV, T. (janvier 2007), La Littérature en péril, Flammarion.

VECK, B. (1994), La Culture littéraire au lycée, des humanités aux méthodes, INRP.

\section{NOTES}

1. Rapport de la COSEF sur la mise en cuvre de la réforme du système d'éducation et de formation (1999-2004).

2. A la rentrée de septembre 2002, les professeurs de français exerçant dans le cycle secondaire qualifiant ont été appelés à mettre en application un nouveau programme conçu autour de la notion de Projet pédagogique ... et l'étude de l'œuvre littéraire intégrale abordée dans son contexte socioculturel. Après une trentaine d'années de règne ininterrompu des "approches communicatives", méthodes d'acquisition exclusivement « fonctionnelle » de la langue et donc d'éviction calculée de toute dimension "culturelle », voilà que l'œuvre littéraire réintègre le cours de français où elle est même invitée à régner en support exclusif !

3. Rapport de la COSEF ...

4. Qui concerne en particulier la maîtrise du français.

5. Publiés par le Ministère de l'Education nationale marocain en 2002.

6. Dans le sens du « bois qui travaille».

7. Mohammed ARKOUN, L'Humanisme arabe au IV/Xe, Paris Vrin, 1970. La Pensée arabe, Paris, PUF, 6e édition, 2003. Humanisme et Islam, Combats et propositions, Librairie philosophique J. VRIN, édition Marsam, 2006. Abdallah LAROUI : La Crise des intellectuels arabes, traditionalisme ou historicisme, Paris, La Découverte, 1974. Islam et Histoire, Albin Michel, 2000. Islam et modernité, La Découverte, 2002.

8. Au sens étymologique du terme : grec sumpatheia, de sun, avec, et pathein, ressentir.

9. Nous pensons aux préparations à l'agrégation de français ou de philosophie.

10. La difficulté, c'est que ce qui doit constituer la plateforme du renouveau est la part la plus fluctuante des domaines de connaissance.

11. Au niveau de la formation des formateurs dans un premier temps.

12. La diversité disciplinaire devrait autoriser des niveaux d'abstraction différents favorisant le recul nécessaire à la perception du caractère inépuisable des jeux taxinomiques.

13. L'immoralisme de Mme Bovary ou la subordination de Meursault à quelque « géocentrisme impérialiste occidental» ...etc. ne sont que des exemples parmi d'autres de blocages moralisateurs.

14. Bulletin officiel n. 41 , novembre 2002, classe de seconde générale et technologique.

15. Journées de formation des professeurs de français, Ministère de l'Éducation Nationale, juin 2002, p. 4.

16. Cette catégorisation est empruntée Par Tzvetan TODOROV à Saint Augustin : ... saint Augustin décrit la différence entre les sentiments que l'on porte à Dieu et aux hommes : de toute chose comme de tout être on peut user en vue d'une fin qui les transcende, de Dieu seul on doit se contenter de jouir. La Littérature en péril, Flammarion, janvier 2007, p. 40.

17. Le mode de vérité qui prévaut actuellement (et depuis peu) du primaire à l'Université est celui découlant des études islamiques! 


\section{RÉSUMÉS}

L'enseignement $d u$ français connaît, depuis près de cinq ans un véritable renouveau. La littérature française a été autorisée à franchir le seuil des collèges et des lycées. Or ce retour s'est effectué dans des conditions telles que sa portée intellectuelle et culturelle semble être d'ores et déjà compromise. La réduction des objectifs de l'enseignement du français à des fonctions platement instrumentales correspond à la volonté d'instaurer un mode de réception cadré, contrôlé, où la libre expression individuelle est bridée.

French teaching knew, five years ago, a real renewal. French litterature has been allowed to go through the threshold of high schools. But, such a return has been done on conditions which compromised the intellectual and the cultural aims. By limiting the goals of French teaching to an instrumental function, there is a real willing to establish a way of learning centred, controlled and putting down individual free speaking.

\section{INDEX}

Mots-clés : enseignement, littérature, culture

Keywords : teaching, literature, culture

\section{AUTEUR}

\section{SOUMYA EL-HARMASSI}

Professeur de l'Enseignement supérieur, École Normale Supérieure de Meknès (Maroc) 